\title{
Joint Allocation of Subcarriers and Transmit Powers in a Multiuser OFDM Cellular Network
}

\author{
Thaya Thanabalasingham ${ }^{\dagger}$, Stephen V. Hanly ${ }^{\dagger}$, Lachlan L. H. Andrew ${ }^{\ddagger}$ and John Papandriopoulos ${ }^{\dagger}$ \\ ${ }^{\dagger}$ ARC Special Centre for Ultra Broadband Information Networks, \\ Department of Electrical and Electronic Engineering, University of Melbourne, Australia \\ \{thaya, hanly, jpap\}@ee.unimelb.edu.au \\ $\ddagger$ Department of Computer Science, California Institute of Technology, California, USA \\ lachlan@caltech.edu
}

\begin{abstract}
In the present paper, we consider the problem of joint bandwidth (subcarriers) and power allocation for the downlink of a multi-user multi-cell OFDM cellular network. This resource allocation problem is formulated as a power minimization problem, subject to meeting the target rates of all users in the network. We develop a distributed solution to find the globally optimal allocation which determines the subcarrier and power allocation dynamically. In addition, we investigate the impact of reducing the complexity by reducing the number of degrees of freedom available in the optimization. In particular, we consider a static bandwidth allocation scheme, and a static power allocation scheme. The numerical results show that the penalty on network performance due to the reduction in the available degrees of freedom is not significant.
\end{abstract}

\section{INTRODUCTION}

Orthogonal Frequency Division Multiplexing (OFDM) is an important technique for communicating over frequency selective, slowly varying channels. The frequency spectrum is divided into orthogonal, non-interfering subcarriers, and different data streams are multiplexed on the different subcarriers. OFDM is well suited to use on frequency selective channels, as it admits very simple equalization. It is both widely applicable, and widely deployed in commercial systems, including in xDSL modems [1], [2], digital video broadcasting [3], and in various wireless systems, including indoor wireless LANs [4]. It is also a strong candidate for $4 \mathrm{G}$ outdoor cellular systems.

Although OFDM typically multiplexes low rate data substreams from a single user onto all the subcarriers, in the downlink of an OFDM cellular network it can also be used to multiplex the data streams from different users onto subsets of the subcarriers. This is the approach taken in the downlink of Flarion's FLASH-OFDM cellular system [5].

This paper considers the resource allocation problem in such a multiple user, multiple cell, OFDM system, and we focus on the downlink, in which the base station is transmitting to mobiles. The users in the same cell do not interfere with each other, but there is "cross-talk" between users in different cells. The resource allocation problem is to decide the number of subcarriers allocated to each user within a cell, and power allocation across the subcarriers. We assume that there are several mobiles in each cell. This may model a wideband cellular OFDM system with full frequency reuse, such as Flarion's system, or a cellular system with aggressive frequency re-use so that co-channel interference is significant.
We consider the case that the average interference experienced by each subcarrier is equal. This may be a result of explicit averaging, such as Flarion's frequency hopping [5], or by constraining the transmit power on each subcarrier to be equal. Although both result in an equal average interference, the instantaneous interference varies in the first case, but remains constant in the second.

As a result of this interference averaging assumption, we only need to model the proportion of subcarriers ("bandwidth") allocated to each mobile and the power spectral density (PSD) for that mobile, but not the exact choice of subcarriers. The total power allocated to a mobile is the product of its PSD and its bandwidth.

In section IV, we seek to minimize the aggregate transmit power of the base stations, subject to meeting all the target data rate requirements of the mobiles. The degrees of freedom per base station are bandwidth and PSD. If there are $L$ mobiles in the cell there are $2 L-1$ degrees of freedom: $L-1$ degrees of freedom in the bandwidth allocation, and $L$ degrees of freedom in the allocation of power spectral densities. We provide a distributed solution to the global optimization problem, which provides an algorithm that each base station can use to find bandwidth and power allocations. Note that by minimizing power, we also maximize the spectral efficiency of the network, as it is an interference limited system.

In Section $\mathrm{V}$, we investigate the impact of reducing the complexity by adding additional constraints. Section V-A considers a "static-subcarriers" scheme, which statically allocates subcarriers in proportion to the rate requirement of each mobile, and then dynamically allocates power to the mobiles. This reduces the $2 L-1$ degrees of freedom to $L$. The resulting algorithm follows Yates' monotonicity framework [6].

Section V-B considers a "uniform PSD" scheme, which constrains the transmit PSD to be the same for all mobiles served by a base station, but optimises over the proportions of subcarriers allocated to each mobile. This again yields $L$ degrees of freedom. This problem is solved by a new dynamic joint bandwidth allocation and power control algorithm.

We then compare the three approaches numerically for a cellular network, with lognormal shadowing and distance loss. Since the free parameters of the approach in section IV are a superset of those in the other approaches, it must have the best performance of the three, and indeed it does. What 
is interesting, however, is how well the other two schemes perform, despite having half as many degrees of freedom. The extra $L-1$ degrees of freedom in section IV buys very little extra capacity.

Of the two simpler schemes, the static-subcarriers scheme has around twice the capacity penalty of uniform PSD scheme. Moreover, the static-subcarriers scheme requires explicit averaging of the interference, whereas this occurs automatically with the uniform PSD scheme.

The static-subcarriers scheme is the traditional approach, taken in the Flarion system [5]. For a given modulation scheme, power control tries to achieve the required signal to interference and noise ratio (SIR) of each mobile. This leads to a near-far problem, and a large dynamic range in the interference levels, requiring explicit interference averaging.

In contrast, in the constant PSD scheme a mobile with a poor SIR can receive more subcarriers. This implicitly allocates the mobile more received power. The downside is the increased requirement for flexible and complex coding and modulation.

\section{RELATED WORK}

Resource allocation in OFDM systems typically uses water pouring [7]. In a multiple user context (such as xDSL links suffering crosstalk) this leads to iterative water-pouring power control methods [8], with the interference between users decreasing as they adapt their signals in the frequency domain to avoid mutual interference. Our formulation avoids this possibility by explicitly whitening the interference as seen by any given mobile, by assuming that each user experiences an average level of interference determined by all the signals for all the mobiles in the nearby cells, not just other-cell mobiles using particular subcarriers allocated to the mobile of interest.

The practical motivation for our approach is provided by the Flarion system [5]. The Flarion system is a wideband OFDM system with frequency hopping to provide frequency diversity, and to average the inter-cell interference, for both the uplink and downlink. The frequency re-use factor is 1 , so low rate codes are used to combat relatively strong levels of intercell interference. Within each cell are orthogonal channels, each consisting of a fast frequency hopping pattern across the subcarriers so as to provide frequency diversity. Mobiles within a cell are allocated one or more channels (depending on their data rate requirement) and thus users are orthogonal within a cell. Each base station is synchronized at the OFDM symbol level, and a design based on Latin squares ensures that during each hopping cycle, any two channels in adjacent cells only use the same subcarrier in the same symbol period exactly once. This provides the interference averaging that we exploit in our analysis. The Flarion system is itself based on earlier work by Wyner [9] which proposed an OFDM wireless system, with full frequency reuse, and frequency hopping to provide interference averaging.

In recent work, [10] formulates a similar problem of minimizing power levels using power and subcarrier allocation, subject to rate constraints on the individual links. There are some key differences, however. On the one hand, [10] addresses frequency selectivity of fading and does not make the interference averaging assumption. On the other hand, their approach is fundamentally combinatorial in nature, and leads to a search for approximate solutions via heuristic algorithms. Also, their approach does not take account of coding. We take a Shannon-theoretic approach, which accounts for coding, and use a relaxed version of the resulting optimization problem that can be solved exactly. Quantization to discrete levels can be applied after the optimal solution is found, and frequency selectivity effects can also be dealt with at the physical layer: a topic for future work.

\section{MODEL}

We consider the resource allocation problem in a multi-cell, multi-user OFDM cellular system focusing on the downlink. The resources are the bandwidth (subcarriers) and power levels, which we consider as providing degrees of freedom for optimization. The main focus of the present paper is to investigate the implication of varying the number of degrees of freedom available to the base stations as it effects network performance.

A cellular network is modeled as a set of $N$ base stations, denoted by $\mathcal{N}=\{1,2, \ldots, N\}$, with each base station $n \in \mathcal{N}$ having a set $C_{n}$ of users. We assume that the number of subcarriers is large compared to the number of users per cell, allowing a continuous allocation of these resources. The apportionment of subcarriers in cell $n$ is modelled by a positive weight vector $\boldsymbol{w}_{n} \in \mathbb{R}_{+}^{L_{n}}$ where $L_{n}=\left|C_{n}\right|$ is the number of users in cell $n$. Each base station is assumed to have the same total number of subcarriers available. A base station $n$ allocates a proportion $w_{n, m}$ of subcarriers to its user $m$. Thus, $\sum_{m \in C_{n}} w_{n, m} \leq 1$. We let $\boldsymbol{w}$ denote the N-tuple of such weight vectors for the network. We note that the optimal weight vector in terms of power minimization will be achieved with an equality, $\sum_{m \in C_{n}} w_{n, m}=1$.

We denote the power density a base station $n$ allocates to user $m \in C_{n}$ by $\rho_{n, m}$. Let $\boldsymbol{\rho}_{n}=\left(\rho_{n, m}\right)_{m \in C_{n}}$ be the vector of power densities in cell $n$. We denote by $\rho$ the N-tuple of such vectors for the network. The power allocated to user $m$ by its base station $n$ is given by $p_{n, m}=w_{n, m} \rho_{n, m}$. Let $\boldsymbol{p}_{n}=\left(p_{n, m}\right)_{m \in C_{n}}$ be the vector of power allocation in cell $n$ and $\boldsymbol{p}=\left(\boldsymbol{p}_{n}\right)_{n \in \mathcal{N}}$ be the N-tuple of such vectors for the network. The total transmit power at base station $n$ is $q_{n}=$ $\sum_{m \in C_{n}} p_{n, m}$. Let $\boldsymbol{q}=\left(q_{n}\right)_{n \in \mathcal{N}}$ be a vector of total powers for the network. If base stations use flat power spectrum across all subcarriers, then, $q_{n}=\rho_{n, m}, \forall m \in C_{n}$ since $\sum_{m \in C_{n}} w_{n, m}=$ 1. See Fig. 1 for an illustration.

We assume that the mobiles experience the average level of interference determined by all mobiles in the interfering cells. Without interference averaging, a user allocated a particular subset of carriers will experience interference from just the signals in these subcarriers from the nearby cells. When the interference on these particular subcarriers is strong, the mobile may require more resources (power and bandwidth) or may not even be able to meet its target rate at all. One way of achieving the effect of interference averaging is to implement some form of signal hopping over subcarriers as in FLASH- 


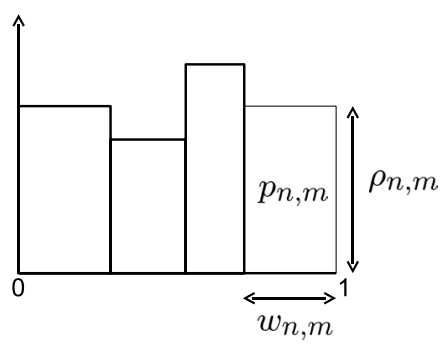

Fig. 1. The power allocation $p_{n, m}$ to user $m \in C_{n}$ depends on power density $\rho_{n, m}$ and the proportion $w_{n, m}$ of bandwidth (subcarriers) allocated to that user, $p_{n, m}=w_{n, m} \rho_{n, m}$. Pictorially, it is the area of the rectangle corresponding to user $m$. The total power $q_{n}$ at base station $n$ is the total area under the curve. With a flat power spectrum, $q_{n}=\rho_{n, m}, \forall m \in C_{n}$ since $\sum_{m \in C_{n}} w_{n, m}=1$.

OFDM. A simpler way is to use a flat transmit power spectrum at the base stations across all subcarriers.

We denote the receiver noise at mobile $m \in C_{n}$ by $\sigma_{m}^{2}>0$. The path gain from a base station $k$ (not necessarily the serving base station of $m$ ) to a user $m$ is $\Gamma_{k, m}$. Then, the signal to interference and noise ratio (SIR) at node $m \in C_{n}$ is given by

$$
\gamma_{n, m}\left(\rho_{n, m}, \boldsymbol{q}\right)=\frac{\Gamma_{n, m} \rho_{n, m}}{\sigma_{m}^{2}+\sum_{k \in \mathcal{N}, k \neq n} \Gamma_{k, m} q_{k}} .
$$

Note that since the bandwidth is taken to be $1, \sigma_{m}^{2}$ and $q_{n}$ are both powers and average spectral densities.

The capacity of a link is determined by both the SIR and the number of subcarriers available. In the present paper, we use the Shannon formula for the bit rate: given the total spectrum allocated to the system is $W \mathrm{~Hz}$, and the SIR is $\gamma$, the maximum bit rate achieved is $W \log _{2}(1+\gamma)$ bits/sec. Since we are using normalized bandwidth, we will use the corresponding notion of spectral efficiency. Thus, if the user is allocated all subcarriers, it has a spectral efficiency of $\log _{2}(1+\gamma)$ bits/sec/Hz. We will assume that mobile $m \in C_{n}$ has a spectral efficiency requirement of $c_{n, m}$. However, the mobile will be allocated a subset of the subcarriers, so the spectral efficiency constraint becomes:

$$
w_{n, m} \log \left(1+\gamma_{n, m}\left(\rho_{n, m}, \boldsymbol{q}\right)\right) \geq c_{n, m} .
$$

\section{Optimal Power and Bandwidth Allocation}

\section{A. Problem Formulation}

We consider here the resource allocation problem of finding optimal power and bandwidth allocation for users. We formulate this resource allocation problem as a power minimization problem, subject to all users achieving their target spectral efficiencies:

$$
\min _{\boldsymbol{\rho}, \boldsymbol{w}} \sum_{n \in \mathcal{N}} \sum_{m \in C_{n}} w_{n, m} \rho_{n, m}
$$

s.t.

$$
\begin{aligned}
w_{n, m} \log \left(1+\gamma_{n, m}\left(\rho_{n, m}, \boldsymbol{q}\right)\right) & \geq c_{n, m}, \forall m \in C_{n}, \\
\sum_{m \in C_{n}} w_{n, m} & =1, \\
\rho_{n, m} & >0, \forall m \in C_{n}, \\
w_{n, m} & >0, \forall m \in C_{n},
\end{aligned}
$$

where $\gamma_{n, m}\left(\rho_{n, m}, \boldsymbol{q}\right)$ is the SIR at user $m$ as defined by (1).

Note that this problem is not convex, but can be transformed into a convex optimization problem by an appropriate change of variables [11]. We do not take this approach here, as it is not clear how to find a distributed solution to the transformed problem.

\section{B. Distributed Solution : Algorithm 1}

In this subsection, we solve the optimization problem (2)(6) by decomposing it into a set of single cell subproblems, each of which is convex. Each subproblem is a problem of allocating subcarriers and power levels within a single cell, assuming the allocations used in other cells remain fixed.

We consider the problem of finding the optimal $\left(\boldsymbol{\rho}_{n}^{*}(\boldsymbol{q}), \boldsymbol{w}_{n}^{*}(\boldsymbol{q})\right)$ for base station $n$ when the vector of total powers for the network, excluding cell $n$, is given by the components of $\boldsymbol{q}$, excluding $q_{n}$. For each $n \in \mathcal{N}$, define $\boldsymbol{\Omega}_{n}$ by

$\boldsymbol{\Omega}_{n}=\left\{\boldsymbol{w}_{n}: \sum_{m \in C_{n}} w_{n, m}=1\right.$ and $\left.w_{n, m} \geq 0, \forall m \in C_{n}\right\}$,

which are the available weight vectors for base station $n$. The optimal $\left(\boldsymbol{\rho}_{n}^{*}(\boldsymbol{q}), \boldsymbol{w}_{n}^{*}(\boldsymbol{q})\right)$ to use at base station $n$ under $\boldsymbol{q}$ will solve the weight-optimization problem:

$$
\min _{\boldsymbol{w}_{n} \in \mathbf{\Omega}_{n}} \sum_{m \in C_{n}} w_{n, m} \rho_{n, m}
$$

subject to meeting constraints (3)-(6).

Rewriting the optimization (7) in terms of $p_{n, m}$ 's, we get:

$$
\min _{\boldsymbol{w}_{n} \in \boldsymbol{\Omega}_{n}} \sum_{m \in C_{n}} p_{n, m}
$$

s.t.

$$
\begin{aligned}
w_{n, m} \log \left(1+\frac{1}{K_{n, m}(\boldsymbol{q})} \frac{p_{n, m}}{w_{n, m}}\right) & \geq c_{n, m}, \quad \forall m \in C_{n}, \\
p_{n, m} & >0, \quad \forall m \in C_{n}, \\
w_{n, m} & >0, \quad \forall m \in C_{n},
\end{aligned}
$$

where

$$
K_{n, m}(\boldsymbol{q})=\frac{\sigma_{m}^{2}+\sum_{k \neq n} \Gamma_{k, m} q_{k}}{\Gamma_{n, m}}
$$

is assumed fixed. Let

$$
g_{n, m}\left(p_{n, m}, w_{n, m}\right)=c_{n, m}-w_{n, m} \log \left(1+\frac{1}{K_{n, m}(\boldsymbol{q})} \frac{p_{n, m}}{w_{n, m}}\right) .
$$

It can be shown that $\nabla^{2} g_{n, m}\left(p_{n, m}, w_{n, m}\right)$ is positive semidefinite. Therefore, the problem (8) is convex in both $\boldsymbol{p}_{n}$ and $\boldsymbol{w}_{n}$. Thus, there exists a unique $\boldsymbol{w}_{n}^{*}(\boldsymbol{q})$ that solves (8). Let the corresponding power allocation be $\boldsymbol{p}_{n}^{*}(\boldsymbol{q})$. The solution to problem (8) can be found using Lagrangian techniques.

Define $I_{n}(\boldsymbol{q})=\sum_{m \in C_{n}} p_{n, m}^{*}(\boldsymbol{q})$. Then, the requirement for base station $n$ to support all of its users is:

$$
q_{n} \geq I_{n}(\boldsymbol{q}) .
$$

The requirement for the network to be able to support the spectral efficiency requirements of all users is given by the vector inequality: 
where $\boldsymbol{I}(\boldsymbol{q})=\left(I_{n}(\boldsymbol{q})\right)_{n \in \mathcal{N}}$.

To show that the solution to the global multi-cell problem (2)-(6) is unique, we apply the framework developed in Yates [6]. Lemma 1 in [6] states that if $\boldsymbol{I}(\boldsymbol{q})$ is standard, and if there is any feasible power vector $\boldsymbol{q}$ satisfying $\boldsymbol{q} \geq \boldsymbol{I}(\boldsymbol{q})$ then $\boldsymbol{I}(\boldsymbol{q})$ has a unique fixed point, which is the minimal solution to the inequality $\boldsymbol{q} \geq \boldsymbol{I}(\boldsymbol{q})$.

A function $\boldsymbol{I}(\boldsymbol{q})$ is standard [6] if for all $\boldsymbol{q} \geq 0$ the following properties are satisfied:

- Positivity $\boldsymbol{I}(\boldsymbol{q})>0$

- Monotonicity If $\boldsymbol{q} \geq \boldsymbol{q}^{\prime}$, then $\boldsymbol{I}(\boldsymbol{q}) \geq \boldsymbol{I}\left(\boldsymbol{q}^{\prime}\right)$

- Scalability For all $\alpha>1, \alpha \boldsymbol{I}(\boldsymbol{q})>\boldsymbol{I}(\alpha \boldsymbol{q})$

Theorem 1: $\boldsymbol{I}(\boldsymbol{q})$ is standard.

Proof:

- Positivity: this follows since $\sigma_{m}^{2}>0$.

- Monotonicity: Suppose that $\boldsymbol{q} \geq \boldsymbol{q}^{\prime}$. When the vector of total powers for the network is $\boldsymbol{q}$, for user $m \in C_{n}$, we have

$$
w_{n, m}^{*}(\boldsymbol{q}) \log \left(1+\frac{1}{K_{n, m}(\boldsymbol{q})} \frac{\left.p_{n, m}^{*}(\boldsymbol{q})\right)}{w_{n, m}^{*}(\boldsymbol{q})}\right)=c_{n, m} .
$$

Now, if instead the interference to mobiles in cell $n$ was generated by the vector $\boldsymbol{q}^{\prime}$, but the bandwidth allocated for user $m$ remains $\boldsymbol{w}_{n}^{*}(\boldsymbol{q})$, let the power allocation at base station $n$ be $\boldsymbol{p}_{n}^{\prime}$, where

$$
w_{n, m}^{*}(\boldsymbol{q}) \log \left(1+\frac{1}{K_{n, m}\left(\boldsymbol{q}^{\prime}\right)} \frac{p_{n, m}^{\prime}}{w_{n, m}^{*}(\boldsymbol{q})}\right)=c_{n, m} .
$$

Using (9) and (10) and the fact that $K_{n, m}(\boldsymbol{q}) \geq$ $K_{n, m}\left(\boldsymbol{q}^{\prime}\right)$, we have $p_{n, m}^{*}(\boldsymbol{q}) \geq p_{n, m}^{\prime}$. Since $\boldsymbol{w}_{n}^{*}(\boldsymbol{q})$ is not necessarily optimal under $\boldsymbol{q}^{\prime}, p_{n, m}^{\prime} \geq p_{n, m}^{*}\left(\boldsymbol{q}^{\prime}\right)$. Hence, $p_{n, m}^{*}(\boldsymbol{q}) \geq p_{n, m}^{*}\left(\boldsymbol{q}^{\prime}\right)$. Consequently, $I_{n}(\boldsymbol{q}) \geq I_{n}\left(\boldsymbol{q}^{\prime}\right)$.

- Scalability: Suppose $\alpha>1$. Let $\boldsymbol{p}_{n}^{\prime}$ be the power allocation at base station $n$ when it uses $\boldsymbol{w}_{n}^{*}(\boldsymbol{q})$ and the vector of total powers for the network is $\alpha \boldsymbol{q}$. Then,

$$
w_{n, m}^{*}(\boldsymbol{q}) \log \left(1+\frac{1}{K_{n, m}(\alpha \boldsymbol{q})} \frac{p_{n, m}^{\prime}}{w_{n, m}^{*}(\boldsymbol{q})}\right)=c_{n, m} .
$$

Using (9) and (11) and the fact that $\alpha K_{n, m}(\boldsymbol{q})>$ $K_{n, m}(\alpha \boldsymbol{q})$, we have $\alpha p_{n, m}^{*}(\boldsymbol{q})>p_{n, m}^{\prime}$. Since $\boldsymbol{w}_{n}^{*}(\boldsymbol{q})$ is not necessarily optimal under $\alpha \boldsymbol{q}, p_{n, m}^{\prime} \geq p_{n, m}^{*}(\alpha \boldsymbol{q})$. Hence, $\alpha p_{n, m}^{*}(\boldsymbol{q})>p_{n, m}^{*}(\alpha \boldsymbol{q})$. Consequently, $\alpha I_{n}(\boldsymbol{q})>$ $I_{n}(\alpha \boldsymbol{q})$. [6],

Since, $\boldsymbol{I}(\boldsymbol{q})$ is standard, the standard power control algorithm

$$
\boldsymbol{q}^{(t+1)}=\boldsymbol{I}\left(\boldsymbol{q}^{(t)}\right)
$$

will converge to the minimal solution. Solving for $I_{n}(\boldsymbol{q})$ at base station $n$ requires the knowledge of $K_{n, m}=\frac{\rho_{n, m}}{\gamma_{n, m}(\boldsymbol{q})}$. Therefore, this algorithm can be executed locally at each base station with the feedback on achieved SIR from only its users, and hence is distributed.

\section{Reduced Complexity Schemes}

With the optimization problem of (2)-(6), the number of degrees of freedom available to base station $n$ is $2 L_{n}-1$ ( $L_{n}$ power densities and $L_{n}-1$ weights) where $L_{n}$ is the number of mobiles in cell $n$. In this section, we consider bandwidth and power allocation problem (2)-(6) with additional constraints. In both schemes that we consider in this section, the additional constraints reduce the number of degrees of freedom available to base station $n$ to $L_{n}$.

\section{A. Per User Power Control : Algorithm 2}

We now consider the resource allocation problem (2)(6) with static bandwidth allocation. The weight vectors are chosen a priori, proportional to the spectral efficiency targets of the users. In cell $n$, the choice of the weight vector is given by:

$$
w_{n, m}=\frac{c_{n, m}}{\sum_{m^{\prime} \in C_{n}} c_{n, m^{\prime}}}, \quad \forall m \in C_{n} .
$$

The resource allocation problem then is to choose the appropriate power spectrum for users with the objective of power minimization, subject to meeting the spectral efficiency requirements of the mobiles. Mathematically, the allocation problem is to solve (2) subject to meeting constraints (3), (5) and (12). The number of degrees of freedom available to base station $n$ is $L_{n}$ : the power density to allocate to each mobile in the cell.

Note that this scheme requires some form of signal hopping over subcarriers, similar to FLASH-OFDM, in order to achieve the effect of interference averaging.

The solution to the resource allocation problem can be found using a similar approach to Section IV-B. Solving the single cell subproblem in this case reduces to solving (3) for power spectral density $\rho_{n, m}^{*}(\boldsymbol{q})$ for each user $m \in C_{n}$ given the weight vector $\boldsymbol{w}_{n}$ (eq. (12)) and the vector of total powers for the network $\boldsymbol{q}$. By defining $I_{n}(\boldsymbol{q})=\sum_{m \in C_{n}} w_{n, m} \rho_{n, m}^{*}(\boldsymbol{q})$, it can be easily shown that this problem falls readily under Yates' framework.

\section{B. Dynamic Bandwidth Allocation : Algorithm 3}

We next consider the resource allocation problem (2)-(6) with the additional constraint that each base station uses a flat transmit power spectrum, that is,

$$
q_{n}=\rho_{n, m}, \quad \forall m \in C_{n} .
$$

Each base station can still choose an appropriate power level and vary the bandwidth allocation to its users. The number of degrees of freedom available to base station $n$ is $L_{n}$, namely: $L_{n}-1$ weights and a single transmit power level. Mathematically, the allocation problem is to solve (2) subject to meeting constraints (3)-(6) and (13).

This problem has been considered in [12] in the context of a general wireless network. A provably convergent distributed algorithm was proposed to solve this problem. The summary of the algorithm is as follows:

Algorithm 3: 
Initialization: Start with any initial transmit power vector $\boldsymbol{q}^{(0)}$. For $k=0,1,2, \ldots$ do:

Iteration $k$ :

- Compute a pseudo-weight $\hat{w}_{n, m}$ for each user $m$ given the the vector of powers for the network, $\boldsymbol{q}^{(k)}$ (using (3)):

$$
\hat{w}_{n, m}=\frac{c_{n, m}}{\log \left(1+\gamma_{n, m}\left(q_{n}^{(k)}, \boldsymbol{q}^{(k)}\right)\right)}, \forall m \in C_{n} .
$$

Define $\hat{\sigma}_{n}=\sum_{m \in C_{n}} \hat{w}_{n, m}$. Note that the pseudo weight vector $\hat{\boldsymbol{w}}_{n}$ computed above is infeasible if $\hat{\sigma}_{n}>1$.

- Compute a feasible weight vector $\boldsymbol{w}_{n}^{(k)}$ by normalizing $\hat{\boldsymbol{w}}_{n}$ :

$$
w_{n, m}^{(k)}=\frac{\hat{w}_{n, m}}{\hat{\sigma}_{n}}, \forall m \in C_{n} .
$$

- Use the newly computed weight vector $\boldsymbol{w}_{n}^{(k)}$ to compute the transmit power requirement $q_{n, m}^{(k)}$ for the users $m \in$ $C_{n}$ (using (3)):

$$
\hat{q}_{n, m}=K_{n, m}\left(\boldsymbol{q}^{(k)}\right)\left[\exp \left(\frac{c_{n, m}}{w_{n, m}^{(k)}}\right)-1\right], \forall m \in C_{n} .
$$

- Compute the power to use for the next iteration depending on the value of $\hat{\sigma}_{n}$ :

$$
q_{n}^{(k+1)}= \begin{cases}\min _{m \in C_{n}} \hat{q}_{n, m}, & \text { if } \hat{\sigma}_{n}>1 \\ \max _{m \in C_{n}} \hat{q}_{n, m}, & \text { otherwise. }\end{cases}
$$

Each iteration of Algorithm 3 can be considered as a mapping $T$ from $\boldsymbol{q}^{(k)}$ to $\boldsymbol{q}^{(k+1)}$. The mapping $T$ is not standard, and hence the results of [6] do not apply. Although $T$ does not satisfy the monotonicity condition required in Yates' framework, the convergence analysis of Algorithm 3 exploits some key properties of the mapping $T$. These properties imply that to any sequence $\left(\boldsymbol{q}^{(k)}\right)_{k=0}^{\infty}$ generated by Algorithm 3, a monotonically non-increasing upper bounding sequence, and a monotonically non-decreasing lower bounding sequence, can be constructed with the property that both bounding sequences provably converge to the minimal solution. See [12] for the details of this argument.

\section{NumericAl Results}

In this section, we numerically evaluate the algorithms developed in this paper. In particular, we investigate the effect on performance in reducing the available degrees of freedom for optimization.

We consider a cellular network that spans an area of $3 \mathrm{~km} \times 3 \mathrm{~km}$. This area is divided into 9 square cells of size $1 \mathrm{~km} \times 1 \mathrm{~km}$. The base stations are located at the center of their respective cells. There are 250 users, uniformly randomly distributed in the network. Each user communicates only to the base station of the cell it is located. The target spectral efficiency for each user is chosen randomly, as described below. We use free-space propagation for distances up to $500 \mathrm{~m}$, and a $\log$ distance path loss, with exponent 3 , beyond that (relative to the reference distance of $500 \mathrm{~m}$ ). For all distances we include a log-normal shadowing with a mean of $0 \mathrm{~dB}$ and a standard deviation of $8 \mathrm{~dB}$. We assume a noise power spectral density of $10^{-19} \mathrm{~W} / \mathrm{Hz}$ at each receiver.

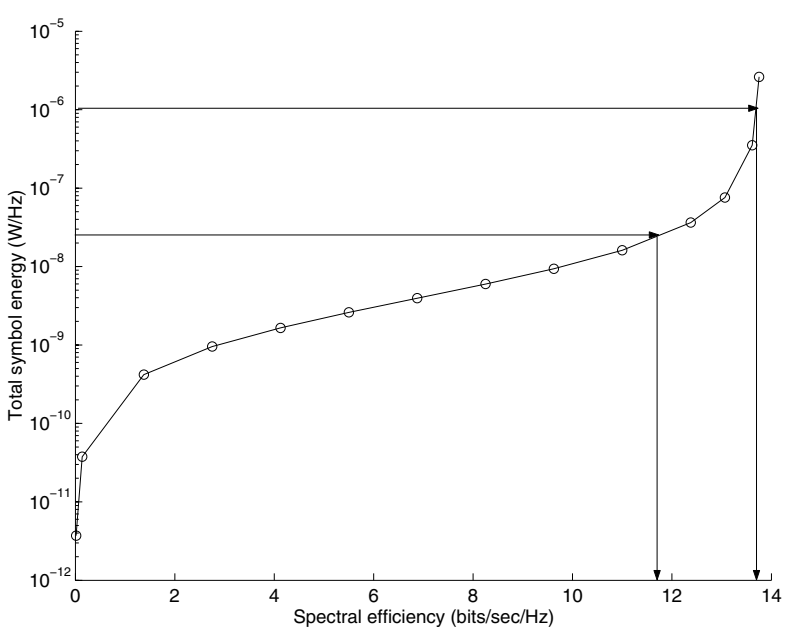

Fig. 2. A particular realization of the network: total symbol energy vs. total spectral efficiency using Algorithm 3

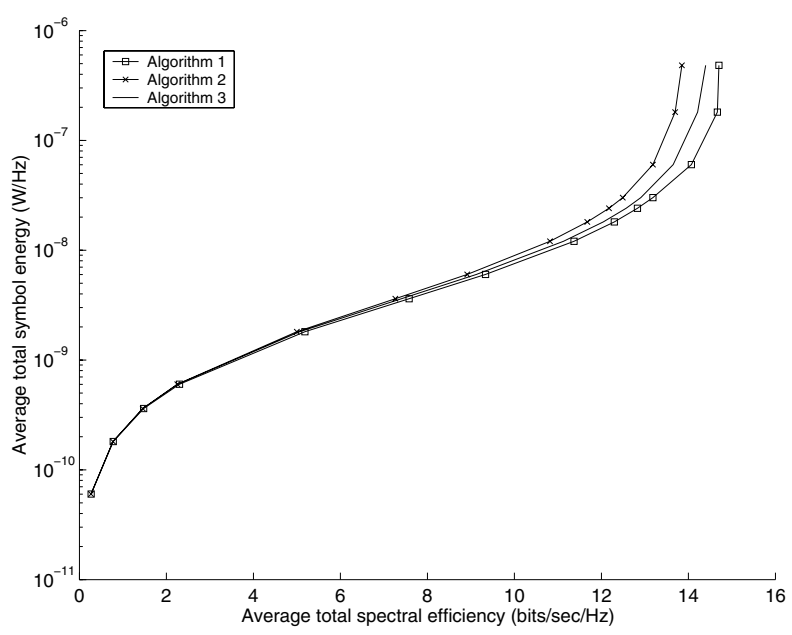

Fig. 3. Average total symbol energy vs. average total spectral efficiency for the network

To obtain the curve of average total symbol energy vs. average total spectral efficiency in Fig. 3, we average total user spectral efficiencies over many random realizations of user locations and shadowing. In each realization of user locations and shadowing, each user selects its target spectral efficiency randomly from a set of spectral efficiencies $\{c, 2 c, 3 c, 4 c\}$, where $c$ is a scaling factor. For each network realization, the spectral efficiencies can be varied by tuning the scaling factor $c$. For each network realization, there is a limit to how high we can scale up $c$; there is an energy explosion that occurs as the capacity limit is reached for that network realization. Fig. 2 depicts the capacity limit for a particular network realization using Algorithm 3. All three algorithms have the same qualitative behaviour.

The averaging of total spectral efficiencies is achieved as follows. For each network realization, the total symbol energies are computed for a set of values of $c$. Then, for a fixed set of total symbol energies, corresponding total spectral efficiencies are computed by interpolation as demonstrated in Fig. 2. The average total spectral efficiency for a given 
total symbol energy is the average of such computed total spectral efficiencies over many network realizations. Fig. 3 plots the average total symbol energy versus average total spectral efficiencies for the three algorithms averaged over 100 realizations. Notice that all three algorithms suffer an energy explosion, as their average capacity limits are reached.

From Fig. 3, it is clear that Algorithm 1 uses the least amount of energy for a given total spectral efficiency. This is not surprising since it solves for the globally optimal allocation. The loss in performance in terms of capacity for a given total symbol energy for Algorithms 2 and 3 is small compared to Algorithm 1 which has all the degrees of freedom available. Of the two reduced complexity schemes, Algorithm 3 marginally outperforms Algorithm 2. The capacity penalty for Algorithm 2 is around twice that of Algorithm 3.

\section{CONCLUSIONS}

We have compared three different power and bandwidth allocation schemes for the downlink of an OFDM cellular system. Algorithm 1 dynamically allocates both subcarriers and power spectral densities to each mobile, and in general the transmit power spectrum of each base station will not be flat. Algorithm 2 allocates subcarriers in a static manner, via (12), and then dynamically allocates power spectral densities to the mobiles. Algorithm 3 allocates subcarriers in a dynamic manner, and allows the base stations to adjust their transmit power dynamically as well, under the constraint that the transmit spectrum is flat. All algorithms solve an optimization problem, in the sense that they provably converge to a solution, when it exists, and when it exists it is unique.

Algorithm 2 incorporates the traditional approach to bandwidth allocation, such as used in the Flarion system, in that the modulation scheme for each mobile is fixed in advance, based on its spectral efficiency requirement. Algorithm 2 is a power control algorithm that tries to ensure that the required SIR's of the mobiles are achieved. In this approach, a mobile close to a base station will receive much less power than a mobile near the cell boundary. As a result, there will be a large dynamic range in the possible interference levels that a mobile will receive from other base stations, since these base stations are also controlling their transmit power levels to accommodate their users. To obtain the interference averaging that we assume in this paper, it is necessary to employ a frequency hopping strategy, and one that is coordinated between cell sites. This is true in the Flarion system, with the Latin square hopping patterns designed to provide the requisite interference averaging (see [5]).

In Algorithm 1, and also Algorithm 3, a mobile far away from the base station can receive more bandwidth (subcarriers) to compensate for the path loss, and this is available also to a mobile experiencing a large amount of interference. Indirectly, allocating more bandwidth will also allocate the mobile more received power, for the same fixed base station transmit power, but there is an additional degree of flexibility in that the modulation scheme can adapt.

With dynamic bandwidth allocation, the mobiles close to the base station will be allocated a small number of subcarriers.
The base station can use a larger constellation size and hence pack more information into the symbols for these users. On the other hand, the users further away from the base station will be allocated a larger number of subcarriers, and hence a higher rate in symbols per second. In this case, only a small constellation size for the symbols is required to achieve the same bit rate. The downside of this approach is the increased complexity of the coding and adaptive modulation required.

From the numerical results section, we conclude that both Algorithm 2 and Algorithm 3, provide solutions that are nearly as good as that of Algorithm 1. Of the two, Algorithm 3 has marginally better performance in terms of power levels or capacity.

The model used in the paper, and in the simulations, takes account of lognormal shadowing and path loss, but not frequency selective multipath fading. With fading, we hope that the solutions provided by the present paper can be retained, with frequency selectivity handled at the lower (physical) layer, but this is a topic for further research. The model is equally applicable to a TDMA system, and in that case, the model already includes flat fading, and the simulation results can easily be extended to include Rayleigh fading.

\section{ACKNOWLEDGEMENT}

This work was supported by the Australian Research Council (ARC).

\section{REFERENCES}

[1] ITU-T Recommendation G.992.1, "Asymmetrical digital subscriber line (ADSL) transceivers," Jul. 1999, also Annex H (10/2000) and Corrigentum $1(11 / 2001)$.

[2] ITU-T Recommendation G.993.1, "Very high-speed digital subscriber line foundation," Nov. 2001.

[3] ETSI EN 300 744, V1.5.1 (2004-11), "Digital video broadcasting (DVB); framing structure, channel coding and modulation for digital terrestrial television (DVB-T)," 2004.

[4] IEEE Std. 802.11g, "Wireless LAN medium access control (MAC) and physical layer (PHY) specifications, amendment 4: Further higher data rate extension in the $2.4 \mathrm{GHz}$ band," Jul. 2003.

[5] D. Tse and P. Viswanath, Fundamentals of Wireless Communication, 1st ed. Cambridge University Press, 2005.

[6] R. D. Yates, "A framework for uplink power control in cellular radio systems," IEEE J. Select. Areas Commun., vol. 13, no. 7, pp. 1341-1347, Sep. 1995

[7] D. J. C. MacKay, Information Theory, Inference, and Learning Algorithms, 1st ed. Cambridge University Press, 2003.

[8] W. Yu, W. Rhee, S. Boyd, and J. M. Cioffi, "Iterative water-filling for Gaussian vector multiple-access channels," IEEE Trans. Info. Theory, vol. 50, no. 1, pp. 145-152, Jan. 2004

[9] A. D. Wyner, "Multi-tone multiple access for cellular systems," AT\&T Bell Labs. Technical Memorandum, BL011217-920812-12TM, 1992.

[10] G. Kulkarni, S. Adlakha, and M. Srivastava, "Subcarrier allocation and bit loading algorithms for OFDMA-based wireless networks," IEEE Trans. Mobile. Comput., vol. 4, no. 6, pp. 652-662, Nov./Dec. 2005.

[11] S. Boyd and L. Vandenberghe, Convex Optimization, 1st ed. Cambridge University Press, 2004.

[12] T. Thanabalasingham, S. V. Hanly, and L. L. H. Andrew, "Joint allocation of transmit power levels and degrees of freedom to links in a wireless network," in Proc. AusCTW, Feb. 2005, pp. 223-229. 